\title{
A Molecular Dynamics Study of the Effect of Voids on the Deformation Behavior of Nanocrystalline Copper
}

\author{
Chen Zheng and Yong-Wei Zhang \\ Department of Materials Science and Engineering, National University of Singapore, Singapore 119260
}

Received 23 June 2007; Revised 11 August 2007; Accepted 24 September 2007

Recommended by Jun Li

Molecular dynamics simulations are performed to study the effect of preexisting ellipsoidal voids on the tensile deformation behavior in nanocrystalline copper. No crack propagation is observed regardless of the orientation of the voids with respect to the tensile direction. However it is found that the voids may assist the shear plane formation via (1) emitting dislocations from the void tips, (2) relieving triple-junction confinement, and (3) catalyzing grain splitting.

Copyright ( $) 2007$ C. Zheng and Y.-W. Zhang. This is an open access article distributed under the Creative Commons Attribution License, which permits unrestricted use, distribution, and reproduction in any medium, provided the original work is properly cited.

\section{INTRODUCTION}

Nanocrystalline materials often show improved mechanical properties such as ultra-high yield and fracture strengths and enhanced superplastic formability at lower temperatures compared to their coarse-grained counterparts [1-5]. On the other hand, the poor sample quality, especially porosity, resulting from the limitation of synthesis techniques often leads to the low values of strength and ductility of nanocrystalline materials [6-10]. For example, experimental results show that the pores in samples made by compaction result in the lack of tensile ductility [7]. The low Young's modulus of nanocrystalline $\mathrm{Pd}$ and $\mathrm{Cu}$ may also be caused by porosity existing in nanocrystalline materials synthesized by inert gas condensation [10]. It is indicated that nanovoids are an intrinsic component of the microstructure of nanocrystalline materials, and even in the dense samples prepared by electrodeposition, nanovoids are still observed [9].

How voids affect the detailed deformation mechanisms of nanocrystalline materials is still largely unclear. Shear marks have been observed on the fracture surfaces of nanocrystalline $\mathrm{Cu}$ in tensile test [11], leading to the conclusion that localized plastic deformation manifested by shear bands can cause an early failure in nanocrystalline materials. Other experimental results also show the formation of shear bands in nanocrystalline $\mathrm{Pd}$ and $\mathrm{Ni}[6,10]$. To understand the shear band formation, a concerted grain boundary sliding model was proposed. Grain boundary sliding, assisted by boundary migration, leads to the plane interface forma- tion $[12,13]$. However, considering the porosity which commonly exists in nanocrystalline materials, the role of voids in the formation and propagation of shear bands may not be ignored. It is anticipated that the presence of voids may lead to the competition between plastic flow and fracture, causing more complicated deformation patterns in a porous crystalline sample.

Previous molecular dynamics simulations mainly focused on nanocrystalline metals without porosity [14-19]. Detailed molecular dynamics simulations of the effect of voids on the deformation behavior of nanocrystalline metals are lacking. Schiotz et al. found that the voids located at grain boundaries had the largest effect on the mechanical properties of nanocrystalline copper [20]. However, they did not reveal how voids affect the deformation patterns of nanocrystalline copper in detail. In this paper, we will investigate the influence of preexisting ellipsoidal voids on the mechanical properties of nanocrystalline copper. The deformation patterns and the evolution of these voids during the deformation will be analyzed. The focus here is on examining whether voids assist the formation and propagation of coordinated shear deformation or cracks and reveals their detailed deformation mechanisms.

\section{SIMULATION PROCEDURE}

A fully dense sample with 125 grains is created first by the Voronoi construction [21] in a cubic box with a side length of $23.1 \mathrm{~nm}$. The total number of atoms in the sample is around 


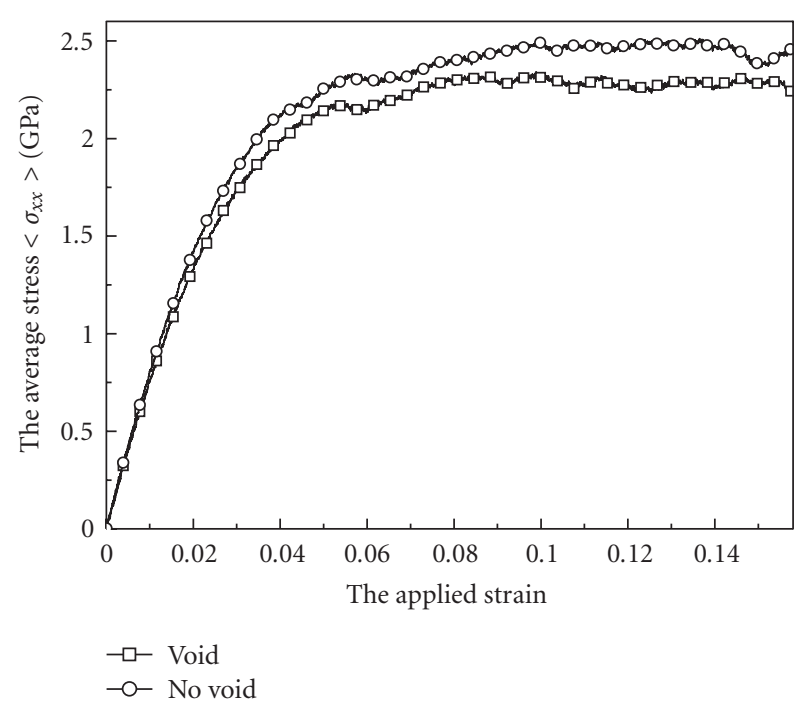

FIGURE 1: The stress-strain curves of the nanocrystalline copper with $2.8 \%$ porosity and without porosity at $300 \mathrm{~K}$.

1 million and the average grain size is $5.8 \mathrm{~nm}$. The orientation of each grain is randomly chosen. Each grain is filled with atoms in an FCC lattice. Then, the system is first annealed under a constant pressure at $300 \mathrm{~K}$ for 50 picoseconds. Subsequently, 42 voids located either in grain boundaries or in the grain interior are introduced, followed by another anneal of 50 picoseconds under a constant pressure at $300 \mathrm{~K}$. The porosity of the sample is about $2.8 \%$. Each void is an oblate ellipsoid with an axis ratio (the ratio of equatorial radius $a$ to the polar radius $c$ ) of 3 . Since it was suggested that pores were comparable in size to the grain size or even smaller $[9,15,22]$, the length of the long axis $2 a$ of the voids varying from $3 \mathrm{~nm}$ to $4.4 \mathrm{~nm}$ is chosen in our simulations. Periodical boundary conditions are applied to the sample. In order to simulate the uniaxial tensile test, the period along the $x$ direction is increased step by step. Since there are no tractions in the lateral directions during uniaxial tensile test, the periods along the $y$ - and $z$-directions are adjusted to ensure the traction-free conditions along the $y$ - and $z$-directions. For comparison, we have performed the simulation of the uniaxial tensile test on its corresponding void-free sample with the same grain structure. The atomic interactions between copper atoms are governed by the embedded atom method (EAM) potential [23] which has been frequently used to simulate the deformation behavior of copper. For comparison, another EAM potential [24] has also been used in the simulations. It is found that the simulation results obtained here are insensitive to the two potentials used. Hence, for brevity, the results presented here are based on [23]. In the above simulations, the applied strain rate is about $5 \times 10^{8} \mathrm{~s}^{-1}$, and the leapfrog method is used in the numerical integration.

Radial distribution functions (RDFs) and common neighbor analysis (CNA) $[25,26]$ are used to analyze the local atomic order of the samples. All atoms in a sample are classified into three classes: FCC, HCP, and "others." The "others" refer to the atoms that are neither in a local FCC nor in a lo- cal HCP environment, and such atoms are mainly located in grain boundaries.

\section{RESULTS AND DISCUSSIONS}

Our simulation shows that the introduction of voids generally weakens the mechanical properties of nanocrystalline materials. An example is given in Figure 1 which shows the stress-strain curves of the samples with $2.8 \%$ porosity and without porosity. It can be seen that both the Young's modulus and the flow stress decrease after the introduction of the voids. The voids result in a $1 \%$ reduction in Young's modulus and a $7.3 \%$ reduction in flow stress. Since the porosity is relatively low (2.8\%), the reduction in Young's modulus and the flow stress is insignificant. These results are consistent with experimental measurements which show that the presence of voids only slightly reduces the values of Young's modulus and moderately reduces tensile strength of nanocrystalline metals $[6,10]$. It should be pointed out that the failure property also depends on the shape of the voids and the deformation mechanisms; if the nanocrystalline materials exhibit brittle behavior without mechanisms available for stress relaxation before fracture, even small levels of porosity may significantly reduce their failure response [27].

The angle between the long axis of an elliptical void and the tensile direction can take any value. We examined the deformation patterns of these voids with various orientations. It is found that even in the most dangerous situation, that is, the long axis of the void is normal to the tensile direction, the void tip still becomes blunt during tensile loading, and no crack propagation from the tip of the voids is observed, indicating a ductile failure mode in the nanocrystalline copper. Figure 2 shows the deformation of a void whose long axis is normal to the tensile direction. It is found that the stress built up at the lower tip of void A during the loading is partially relieved by intragranular slips through the emission of dislocations, causing blunting of the void tips. The dislocation emission can be clearly seen in grain 26 of Figure 2(b) at a strain level of $16.8 \%$. We have also examined the stress distributions before and after the dislocation emission, which is at about $12 \%$ strain. Due to the dislocation emissions, the void tip becomes blunt and the stress relief around the tip of the void occurs. Thus, a certain level of ductility in nanocrystalline copper is expected in the presence of voids. The reason for the ductility may be due to what follows. First, the small grain size (an average of $5.8 \mathrm{~nm}$ ) leads to a small void size, which increases the difficulties for stress concentration to be built up. Second, even if stress is built up at the void tips, the emission of dislocations relieves the stress concentration. Both factors suppress the crack propagation.

Although no crack nucleation and propagation from voids have been observed in our simulations, it is found that the formation and propagation of shear planes are closely related to the presence of voids. Here, a shear plane refers to a coordinated shear deformation across several grains. These shear planes can be considered as precursors for the formation of shear bands. Figure 2 demonstrates the formation of a shear plane (shear plane 1) by showing an X-Z slice of the sample. The FCC, HCP, and grain boundary atoms are 


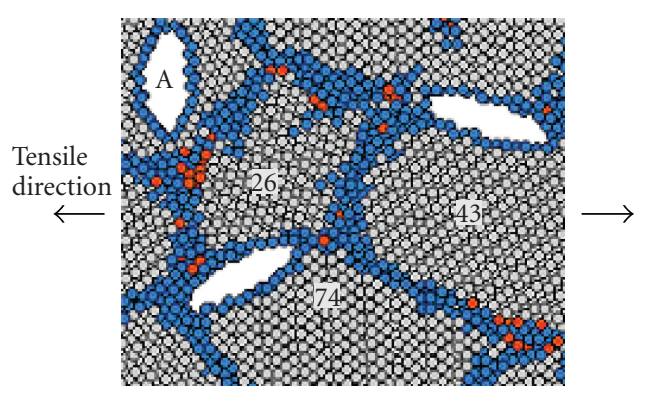

(a)

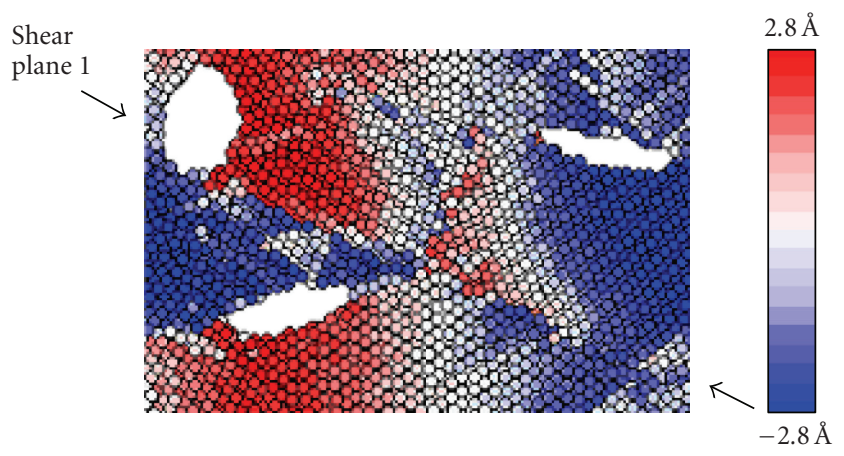

(c)

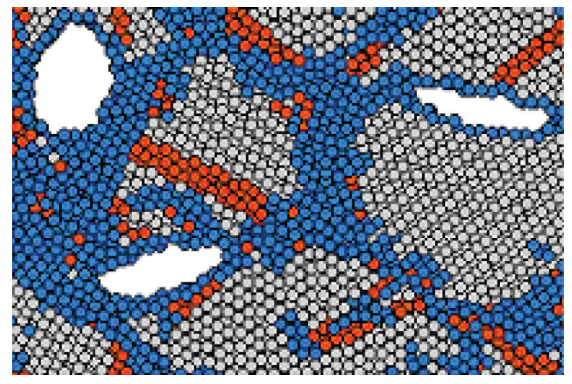

(b)

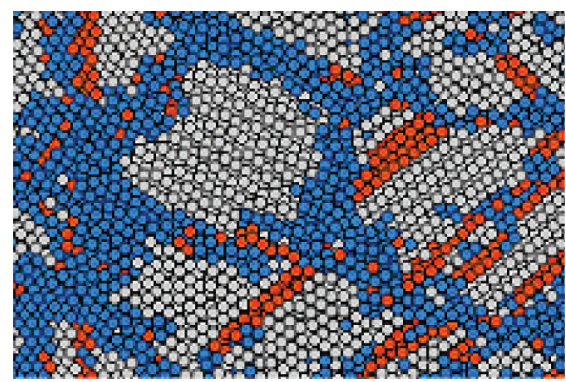

(d)

FIGURE 2: A region of the nanocrystalline copper at different deformation levels. (a) The atomic configuration of the porous sample at $0 \%$ strain; (b) the atomic configuration of the porous sample at $16.8 \%$ strain; (c) the relative displacement of the atomic motion in the $x$-direction to the uniform deformation of the porous sample at $16.8 \%$ strain; (d) the atomic configuration of the sample without voids at $16.8 \%$ strain. In (c), the arrows display shear plane 1.

shown in grey, red, and blue, respectively. Initially, the sample is free of dislocations, and only some HCP clusters in the grain boundaries are observed as shown in Figure 2(a). An intragranular slip caused by partial dislocations in grain 26 after $16.8 \%$ deformation can be seen in Figure 2(b). These partial dislocations are emitted from the lower tip of void A which is located near a triple junction (TJ) region. Subsequently, a grain boundary sliding between grain 43 and grain 74 occurs to form shear plane 1. Apparently, the emission of dislocations from void tips assists the formation of shear planes. To visualize shear planes, the relative motion of the atoms in the $x$-direction (tensile direction) to the uniform deformation is shown in Figure 2(c). The atoms marked by red move relatively to the right and the atoms marked by blue move relatively to the left. The magnitude of displacement is indicated by the color bar in Figure 2(c) (c). The change of color in the two sides of the shear plane clearly indicates the coordinated shear behavior. We have also examined the relative motion of the atoms in the $z$-direction; an opposite movement of atoms across the shear plane 1 is also observed. In order to reveal the role of voids, the atomic configuration of the sample without voids at $16.8 \%$ deformation is also displayed in Figure 2(d). It can be seen that no slip plane goes through grain 26 , highlighting the assisting role of voids in the formation of shear plane.

Figure 3 shows the formation of shear plane 2 in another region of the porous sample. The atomic configura- tions at the strain levels of $0 \%$ and $13.2 \%$ are shown in Figures 3(a) and 3(b), respectively. Voids D, E, F, and G are located at triple junctions and the other two voids are along grain boundaries. From Figure 3(c), it can be seen that the formation of shear plane 2 is a process that involves an intragranular slip in grain 70 , which is induced by void $\mathrm{F}$, together with concerted grain boundary slidings between grains 20 and 15, between grains 97 and 69, and between grains 5072. It is noted that the migration of the triple junction between grains 20,69, and 97 allows for the formation of shear plane 2. More importantly, as voids D and E release the geometry confinement caused by the triple junction, both the alignment of these grain boundaries and the grain boundary slidings between grains 97 and 69 and between grains 50 and 72 occur to form shear plane 2 as shown in Figure 3(c). No such alignment of grain boundaries between grains 97 and 69 and between grains 50 and 72 is observed in the void-free sample (see Figure 3(d); circles denote the associated triple junction regions). Therefore, there is no shear plane between grains 97 and 69 and grains 50 and 72 in the void-free sample.

Figure 4 shows another section of the porous sample with a different shear plane (shear plane 3 ). Initially, the two voids are located along the grain boundaries between grains 21 and 45 and between grains 49 and 54, respectively, and separated by grain 96 as shown in Figure 4(a). After 14.4\% deformation, it can be seen from Figure 4(b) that the two voids 


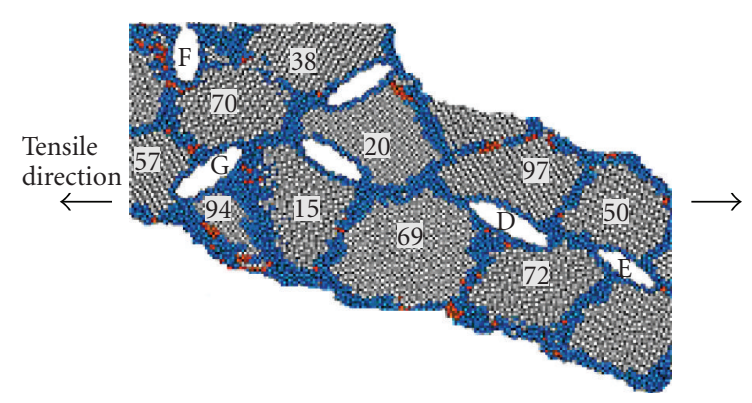

(a)

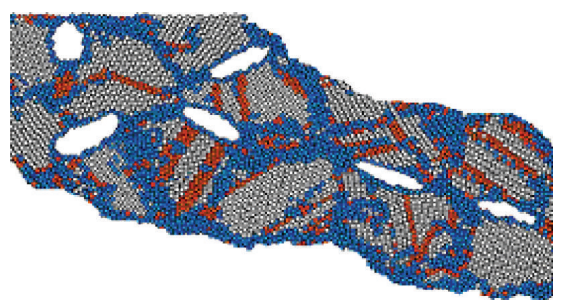

(b)

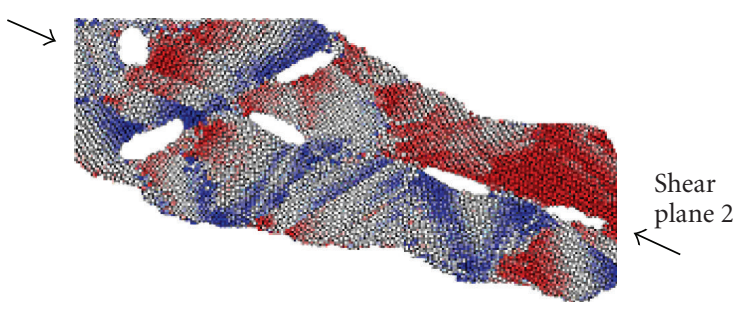

(c)

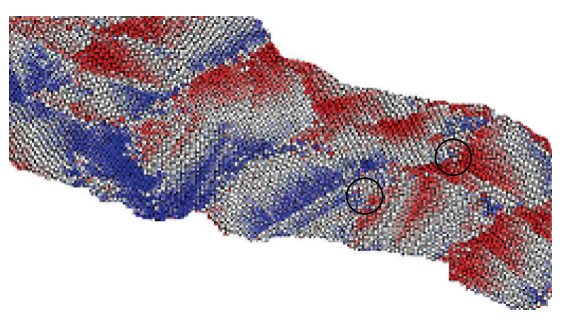

(d)

FIGURE 3: A region of the nanocrystalline copper at different deformation levels. (a) The atomic configuration of the porous sample at $0 \%$ strain; (b) the atomic configuration of the porous sample at $13.2 \%$ strain; (c) the relative displacement of the atomic motion in the $x$-direction to the uniform deformation of the porous sample at $13.2 \%$ strain; (d) the relative displacement of the atomic motion in the $x$-direction to the uniform deformation of the sample without void at $13.2 \%$ strain. In (c), the arrows display shear plane 2 .

expand and a new grain boundary connecting the two voids appears within grain 96 . The driving force for the splitting of grain 96 into two grains may be associated with the expansion of the two voids. Figure 4(c) shows the relative atomic displacement in the $x$-direction at a $14.4 \%$ strain. It is noted that in the nearby region of shear plane 3 , atoms in grain 45 slide are relative to atoms in grain 21 . Similarly, atoms in the new grain (the right part of grain 96) move relative to atoms in the other new grain (the left part of grain 96). Atoms in grain 54 also move relative to atoms in grain 49 . These synergetic motions of several grains cause the formation of shear plane 3. Only dislocation activities are observed in the voidfree sample and no new grain formation is observed even after a $14.4 \%$ deformation as shown in Figure 4(d). It is found that all the observed shear planes are orientated at 30$65^{\circ}$ away from the tensile axis, which is qualitatively consistent with the experimental results on nanocrystalline metals $[10,11]$.

MD simulations without nanovoids have showed that there are three mechanisms contributing to the formation of shear planes [15, 16]: (1) GB sliding-induced migration to form a single shear plane consisting of a number of collinear GBs, (2) the coalescence via reorientation of neighboring grains, and (3) continuity of the shear plane by intragranular slip. Our simulation has shown that the presence of nanovoids facilitates the formation of shear planes in the deformation of nanocrystalline copper. At very small scale (one grain size), the existence of voids gives rise to the nanoscale localized plastic deformation through void tip blunting (emission of dislocations). At larger scale (size of several grains), since the nanovoids are preferentially located at grain boundaries or triple junctions, the presence of voids assists the shear plane formation by releasing the constraints around the voids. In addition, our simulation also shows that a shear plane can extend via the formation of a new grain boundary by splitting one grain into two. This process is facilitated by the shrinkage and expansion of void volume (rearrangement of atoms). Both present simulation and previous simulations show that there is a strong propensity for shear plane formation in nanocrystalline materials. Several experimental observations have also shown the evidence of shear deformation characteristics. For example, the tensile test results of nanocrystalline copper exhibit a clear sign of plasticity at the nanoscale which takes the form of lineshaped markings similar to persistent shear bands [10]. In addition, very narrow shear band formation has also been observed in other nanocrystalline materials $[6,11]$. For the results presented here, fracture by cracking is not observed even at large strain (13\%). This unreasonable high ductility may be caused by the periodic boundary conditions used in the present simulations. Since there are no spatial confinements in real tensile test, the strong propensity for the shear band formation in nanocrystalline materials may lead to a much earlier failure.

\section{CONCLUSIONS}

Our simulations have shown that both the values of Young's modulus and the flow stress of the nanocrystalline copper with porosity are lower than those without porosity. Preexisting ellipsoidal voids become blunt during the deformation and no crack-like void propagation along grain boundaries is observed. It is shown that the formation of a shear plane can be assisted by voids. The assisting mechanisms can be summarized as follows: (1) the intragranular slip caused by the emission and propagation of dislocations from the tips of voids, (2) the released geometry confinement by a void located at a triple junction, together with triple junction 


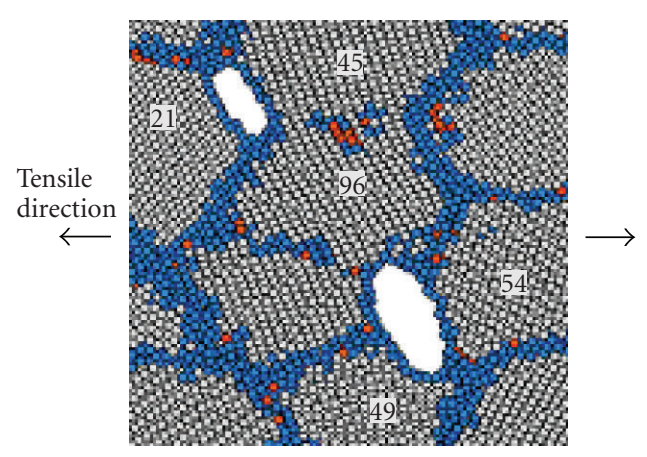

(a)

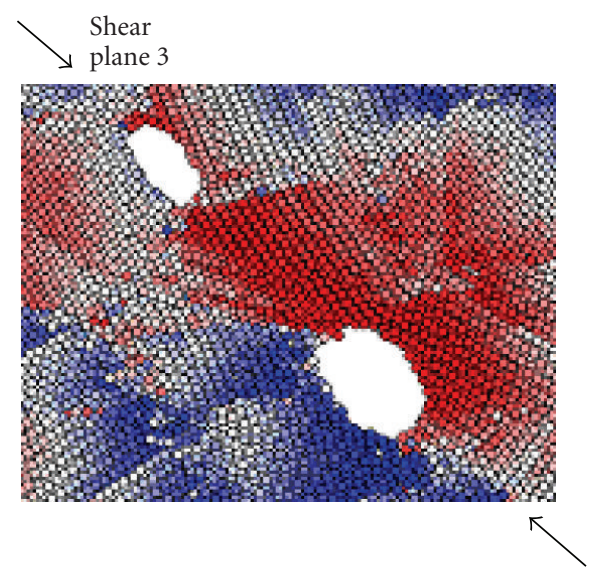

(c)

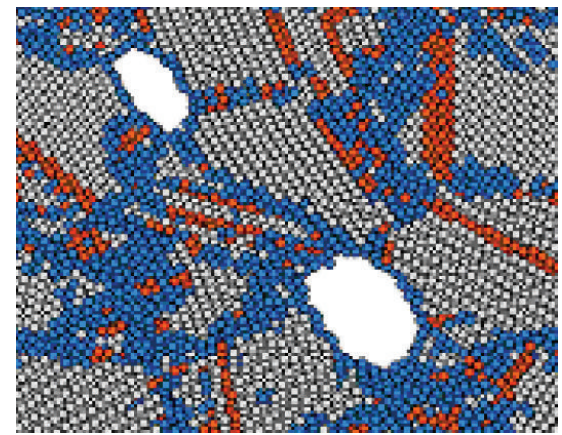

(b)

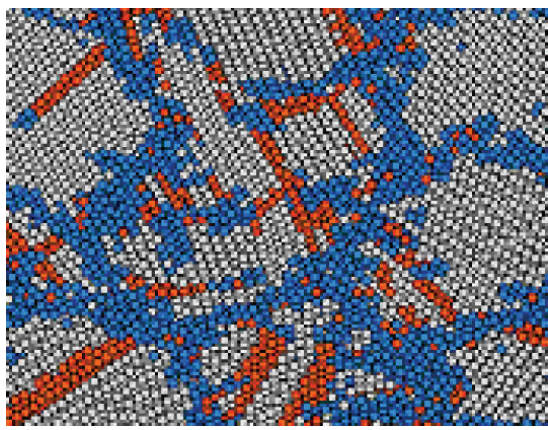

(d)

FIGURE 4: A region of the nanocrystalline copper at different deformation levels. (a) The atomic configuration of the porous sample at $0 \%$ strain; (b) the atomic configuration of the porous sample at $14.4 \%$ strain; (c) the relative displacement of the atomic motion in the $x$-direction to the uniform deformation of the porous sample at $14.4 \%$ strain; (d) the atomic configuration of the sample without voids at $14.4 \%$ strain. In (c), the arrows display shear plane 3.

migration and grain rotation to facilitate the alignment of grain boundaries, (3) extending a shear plane by the grain boundary formation through the splitting of one grain into two grains.

\section{REFERENCES}

[1] H. Gleiter, "Nanocrystalline materials," Progress in Materials Science, vol. 33, no. 4, pp. 223-315, 1989.

[2] C. Suryanarayana, "Nanocrystalline materials," International Materials Reviews, vol. 40, no. 2, pp. 41-64, 1995.

[3] D. H. Jeong, F. Gonzalez, G. Palumbo, K. T. Aust, and U. Erb, "The effect of grain size on the wear properties of electrodeposited nanocrystalline nickel coatings," Scripta Materialia, vol. 44, no. 3, pp. 493-499, 2001.

[4] R. W. Siegel, "Mechanical properties of nanophase materials," Materials Science Forum, vol. 235-238, pp. 851-860, 1997.

[5] L. Lu, M. L. Sui, and K. Lu, "Superplastic extensibility of nanocrystalline copper at room temperature," Science, vol. 287, no. 5457, pp. 1463-1466, 2000.

[6] P. G. Sanders, C. J. Youngdahl, and J. R. Weertman, "The strength of nanocrystalline metals with and without flaws," Materials Science and Engineering A, vol. 234-236, pp. 77-82, 1997.
[7] P. G. Sanders, J. A. Eastman, and J. R. Weertman, "Elastic and tensile behavior of nanocrystalline copper and palladium," Acta Materialia, vol. 45, no. 10, pp. 4019-4025, 1997.

[8] S. R. Agnew, B. R. Elliott, C. J. Youngdahl, K. J. Hemker, and J. R. Weertman, "Microstructure and mechanical behavior of nanocrystalline metals," Materials Science and Engineering A, vol. 285, no. 1-2, pp. 391-396, 2000.

[9] S. van Petegem, F. Dalla Torre, D. Segers, and H. van Swygenhoven, "Free volume in nanostructured Ni," Scripta Materialia, vol. 48, no. 1, pp. 17-22, 2003.

[10] P. G. Sanders, J. R. Weertman, and J. G. Barker, "Structure of nanocrystalline palladium and copper studied by small angle neutron scattering," Journal of Materials Research, vol. 11, no. 12, pp. 3110-3120, 1996.

[11] Y. M. Wang, K. Wang, D. Pan, K. Lu, K. J. Hemker, and E. $\mathrm{Ma}$, "Microsample tensile testing of nanocrystalline copper," Scripta Materialia, vol. 48, no. 12, pp. 1581-1586, 2003.

[12] H. Hahn and K. A. Padmanabhan, "A model for the deformation of nanocrystalline materials," Philosophical Magazine B, vol. 76, no. 4, pp. 559-571, 1997.

[13] K. A. Padmanabhan, G. P. Dinda, H. Hahn, and H. Gleiter, "Inverse Hall-Petch effect and grain boundary sliding controlled flow in nanocrystalline materials," Materials Science and Engineering A, vol. 452-453, pp. 462-468, 2007. 
[14] Y. W. Zhang, P. Liu, and C. Lu, "Molecular dynamics simulations of the preparation and deformation of nanocrystalline copper," Acta Materialia, vol. 52, no. 17, pp. 5105-5114, 2004.

[15] A. Hasnaoui, H. van Swygenhoven, and P. M. Derlet, "Dimples on nanocrystalline fracture surfaces as evidence for shear plane formation," Science, vol. 300, no. 5625, pp. 1550-1552, 2003.

[16] A. Hasnaoui, H. van Swygenhoven, and P. M. Derlet, "Cooperative processes during plastic deformation in nanocrystalline fcc metals: a molecular dynamics simulation," Physical Review B, vol. 66, no. 18, Article ID 184112, 8 pages, 2002.

[17] J. Schiøtz, F. D. Di Tolla, and K. W. Jacobsen, "Softening of nanocrystalline metals at very small grain sizes," Nature, vol. 391, no. 6667, pp. 561-563, 1998.

[18] H. van Swygenhoven, A. Caro, and D. Farkas, "A molecular dynamics study of polycrystalline fcc metals at the nanoscale: grain boundary structure and its influence on plastic deformation," Materials Science and Engineering A, vol. 309-310, pp. 440-444, 2001.

[19] H. van Swygenhoven, "Grain boundaries and dislocations," Science, vol. 296, no. 5565, pp. 66-67, 2002.

[20] J. Schiøtz, T. Vegge, F. D. Di Tolla, and K. W. Jacobsen, "Atomic-scale simulations of the mechanical deformation of nanocrystalline metals," Physical Review B, vol. 60, no. 17, pp. 11971-11983, 1999.

[21] G. Z. Voronoi, "Nouvelles applications des paramtres continus $\mathrm{s}$ la thorie des. Formes quadratiques, deuxime mmoire," Journal für die Reine und Angewandte Mathematik, vol. 134, pp. 198-287, 1908.

[22] P. G. Sanders, J. A. Eastman, and J. R. Weertman, "Pore distributions in nanocrystalline metals from small-angle neutron scattering," Acta Materialia, vol. 46, no. 12, pp. 4195-4202, 1998.

[23] M. W. Finnis and J. E. Sinclair, "A simple empirical N-body potential for transition metals," Philosophical Magazine A, vol. 50, no. 1, pp. 45-55, 1984.

[24] J. Cai, "The structural phase transformation and elastic instability in F.C.C. metals," Physica Status Solidi B, vol. 203, no. 2, pp. 345-356, 1997.

[25] J. D. Honeycutt and H. C. Andersen, "Molecular dynamics study of melting and freezing of small Lennard-Jones clusters," Journal of Physical Chemistry, vol. 91, no. 19, pp. 4950-4963, 1987.

[26] A. S. Clarke and H. Jónsson, "Structural changes accompanying densification of random hard-sphere packings," Physical Review E, vol. 47, no. 6, pp. 3975-3984, 1993.

[27] V. Krstic, U. Erb, and G. Palumbo, "Effect of porosity on Young's modulus of nanocrystalline materials," Scripta Metallurgica et Materialia, vol. 29, no. 11, pp. 1501-1504, 1993. 

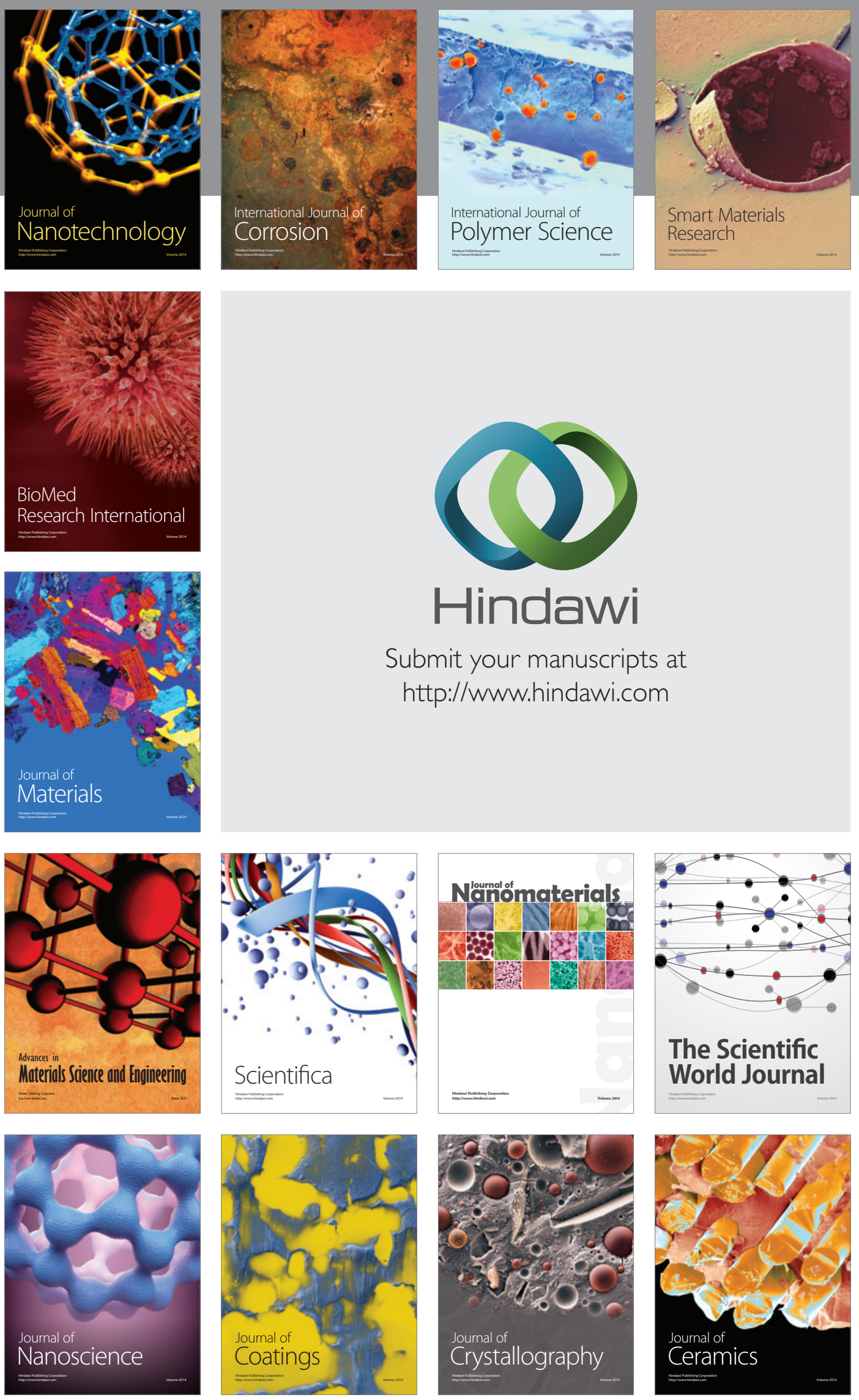

The Scientific World Journal

Submit your manuscripts at

http://www.hindawi.com

\section{World Journal}

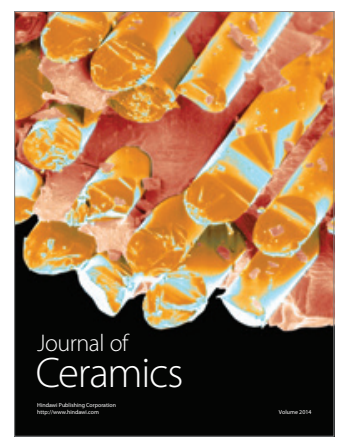

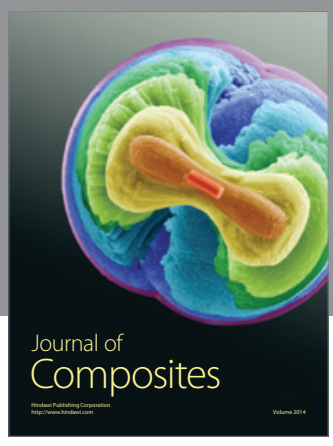
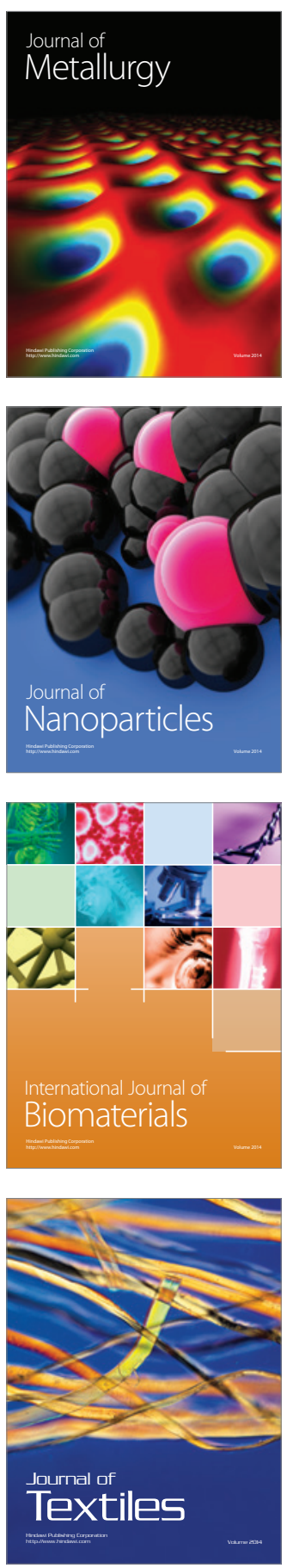